\title{
Growth and poles of solutions of systems of complex composite functional equations
}

\author{
Hua Wang ${ }^{1 *}$, Yong Huang ${ }^{2}$ and Hong-Yan $\mathrm{Xu}^{1}$
}

"Correspondence:

hhhlucy2012@126.com

${ }^{1}$ Department of Informatics and Engineering, Jingdezhen Ceramic

Institute, Jingdezhen, Jiangxi 333403, China

Full list of author information is available at the end of the article

\begin{abstract}
In this paper, we investigate the growth of transcendental meromorphic solutions of some types of systems of complex functional equations and obtain the lower bounds for Nevanlinna lower order for meromorphic solutions of such equations. Our results are improvement of the previous theorems given by Gao, Zheng and Chen. Some examples are also given to illustrate our results.
\end{abstract}

MSC: 39A50; 30D35

Keywords: system; $q$-shift; difference equation

\section{Introduction and main results}

Throughout this paper, the term 'meromorphic' will always mean meromorphic in the complex plane $\mathbb{C}$. Considering a meromorphic function $f$, we shall assume that readers are familiar with the fundamental results and the standard notations of the Nevanlinna value distribution theory of meromorphic functions such as $m(r, f), N(r, f), T(r, f)$, the first and second main theorems, lemma on the logarithmic derivatives etc. of Nevanlinna theory (see Hayman [1], Yang [2] and Yi and Yang [3]). We also use $\rho(f), \mu(f), \lambda(f)$ and $\lambda\left(\frac{1}{f}\right)$ to denote the order, the lower order, the exponent of convergence of zeros and the exponent of convergence of poles of $f(z)$, respectively, and $S(r, f)$ to denote any quantity satisfying $S(r, f)=o(T(r, f))$ for all $r$ outside a possible exceptional set of finite logarithmic measure $\lim _{r \rightarrow \infty} \int_{[1, r) \cap E} \frac{d t}{t}<\infty$.

Recently, there have been a number of papers focusing on the growth of solutions of difference equations, value distribution and uniqueness of differences analogues of Nevanlinna's theory (including [4-9]). Based on these results given in [10-12], people obtained many interesting theorems in the fields of complex analysis.

In 2003, Silvennoinen [13] studied the growth and existence of meromorphic solutions of functional equations of the form $f(p(z))=R(z, f(z))$ and obtained the following result.

Theorem 1.1 [13] Let $f$ be a non-constant meromorphic solution of the equation

$$
f(p(z))=R(z, f(z))=\frac{\sum_{i=0}^{m_{1}} a_{i}(z) f(z)^{i}}{\sum_{j=0}^{n_{1}} b_{j}(z) f(z)^{j}}
$$

where $p(z)$ is an entire function, $a_{i}, b_{j}$ are small meromorphic functions with respect to $f$. Then $p(z)$ is a polynomial.

02013 Wang et al.; licensee Springer. This is an Open Access article distributed under the terms of the Creative Commons Attribution License (http://creativecommons.org/licenses/by/2.0), which permits unrestricted use, distribution, and reproduction in any medium, provided the original work is properly cited. 
In 2012, Gao [14] studied the problem when the above equation is replaced by the following system of function equations:

$$
\left\{\begin{array}{l}
f_{1}(p(z))=R_{1}\left(z, f_{2}(z)\right)=\frac{\sum_{i=0}^{m_{1}} a_{i}(z) f_{2}(z)^{i}}{\sum_{j=0}^{n_{1}} b_{j}(z) f_{2}(z)^{j}}, \\
f_{2}(p(z))=R_{2}\left(z, f_{1}(z)\right)=\frac{\sum_{i=0}^{m_{1}} c_{i}(z) f_{1}(z)^{i}}{\sum_{j=0}^{n_{1}} d_{j}(z) f_{1}(z)^{j}},
\end{array}\right.
$$

where $p(z)$ is an entire function, $R_{1}\left(z, f_{2}(z)\right), R_{2}\left(z, f_{1}(z)\right)$ are irreducible rational functions, the coefficients are small functions; and he obtained the following.

Theorem 1.2 [14, Theorem 1] Let $\left(f_{1}, f_{2}\right)$ be a non-constant meromorphic solution of system (1). Then $p(z)$ is a polynomial.

After his works, Gao [15, 16], Xu et al. [17] further investigated the growth and existence of meromorphic solutions of some types of systems of complex functional equations and obtained a series of results (see $[15,16,18,19]$ ). Inspired by the ideas of Refs. [14-16, 20,21 ], we investigate some properties of solutions of some types of systems of complex functional equations and obtain the following results.

The first theorem is about meromorphic solutions with few zeros and poles of a type of system of complex functional equations.

Theorem 1.3 Let $c_{j} \in \mathbb{C} \backslash\{0\}$ and suppose that $f_{1}, f_{2}$ are a pair of non-rational meromorphic solutions of the system

$$
\left\{\begin{array}{l}
\prod_{j=1}^{n_{1}} f_{1}\left(z+c_{j}\right)=\frac{a_{0}(z)+a_{1}(z) f_{2}(z)+\cdots+a_{p_{2}}(z) f_{2}(z)^{p_{2}}}{b_{0}(z)+b_{1}(z) f_{2}(z)+\cdots+b_{q_{2}}(z) f_{2}(z)^{q_{2}}}, \\
\prod_{j=1}^{n_{2}} f_{2}\left(z+c_{j}\right)=\frac{e_{0}(z)+e_{1}(z) f_{1}(z)+\cdots+e_{p_{1}}(z) f_{1}(z)^{p_{1}}}{d_{0}(z)+d_{1}(z) f_{1}(z)+\cdots+d_{q_{1}}(z) f_{1}(z)^{q_{1}}},
\end{array}\right.
$$

with the coefficients $a_{i}(z), b_{i}(z), e_{i}(z), d_{i}(z)$ being small functions with respect to $f_{1}, f_{2}$ and $a_{p_{2}}(z) b_{q_{2}}(z) e_{p_{1}}(z) d_{q_{1}}(z) \not \equiv 0$. If

$$
\max \left\{\lambda\left(f_{t}\right), \lambda\left(1 / f_{t}\right)\right\}<\rho\left(f_{t}\right), \quad t=1,2,
$$

then system (2) is of the form

$$
\left\{\begin{array}{l}
\prod_{j=1}^{n_{1}} f_{1}\left(z+c_{j}\right)=c_{2}(z) f_{2}(z)^{s_{2}}, \\
\prod_{j=1}^{n_{2}} f_{2}\left(z+c_{j}\right)=c_{1}(z) f_{1}(z)^{s_{1}}
\end{array}\right.
$$

where $c_{1}(z), c_{2}(z)$ are meromorphic functions, $T\left(r, c_{1}\right)+T\left(r, c_{2}\right)=S\left(r, f_{1}\right)+S\left(r, f_{2}\right), s_{1}, s_{2} \in \mathbf{Z}$.

Theorem 1.4 Suppose that $\left(f_{1}, f_{2}\right)$ are a pair of transcendental meromorphic solutions of the system of q-shift difference equations

$$
\left\{\begin{array}{l}
\sum_{j=1}^{n} a_{j}^{1}(z) f_{1}\left(q^{j} z+c_{j}\right)=\sum_{i=0}^{d_{1}} b_{i}^{1}(z) f_{2}(z)^{i}, \\
\sum_{j=1}^{n} a_{j}^{2}(z) f_{2}\left(q^{j} z+c_{j}\right)=\sum_{i=0}^{d_{2}} b_{i}^{2}(z) f_{1}(z)^{i},
\end{array}\right.
$$


where $c_{j} \in \mathbb{C} \backslash\{0\}, q \in \mathbb{C},|q|>1, d_{1} d_{2} \geq 2$ and the coefficients $a_{j}^{t}(z), b_{i}^{t}(z)(t=1,2)$ are rational functions. If $f_{t}(t=1,2)$ are entire or have finitely many poles, then there exist constants $K_{t}>0(t=1,2)$ and $r_{0}>0$ such that for all $r \geq r_{0}$,

$$
\log M\left(r, f_{t}\right) \geq K_{t}\left(d_{1} d_{2}\right)^{\frac{\log r}{2 n \log |q|}, \quad t=1,2 .}
$$

Theorem 1.5 Suppose that $\left(f_{1}, f_{2}\right)$ are a pair of transcendental meromorphic solutions of the system of $q$-shift difference equations

$$
\left\{\begin{array}{l}
\sum_{j=1}^{n_{1}} a_{j}^{1}(z) f_{1}\left(q^{j} z+c_{j}\right)=\frac{P_{2}\left(z f_{2}(z)\right)}{Q_{2}\left(z f_{2}(z)\right)}, \\
\sum_{j=1}^{n_{2}} a_{j}^{2}(z) f_{2}\left(q^{j} z+c_{j}\right)=\frac{P_{1}\left(z f_{1}(z)\right)}{Q_{1}\left(z f_{1}(z)\right)},
\end{array}\right.
$$

where $c_{j} \in \mathbb{C} \backslash\{0\}, q \in \mathbb{C},|q|>1$, the coefficients $a_{j}^{t}(z), t=1,2$, are rational functions, and $P_{t}, Q_{t}$ are relatively prime polynomials in $f_{t}$ over the field of rational functions satisfying $p_{t}=\operatorname{deg}_{f_{t}} P_{t}, l_{t}=\operatorname{deg}_{f_{t}} Q_{t}, d_{t}=p_{t}-l_{t} \geq 2, t=1$, 2. If $f_{t}(t=1,2)$ have infinitely many poles, then for sufficiently large $r$,

$$
n\left(r, f_{t}\right) \geq K_{t}\left(d_{1} d_{2}\right)^{\frac{\log r}{\left(n_{1}+n_{2}\right) \log |q|}}, \quad t=1,2,
$$

and

$$
\mu\left(f_{1}\right)+\mu\left(f_{2}\right) \geq \frac{2\left(\log d_{1}+\log d_{2}\right)}{\left(n_{1}+n_{2}\right) \log |q|} .
$$

Remark 1.1 Since system (4) is a particular case of system (5), from the conclusions of Theorem 1.5, we can get the following result.

Under the assumptions of Theorem 1.4. If $f_{t}(t=1,2)$ have infinitely many poles, then there exist constants $K_{t}>0(t=1,2)$ and $r_{0}>0$ such that for all $r \geq r_{0}$,

$$
n\left(r, f_{t}\right) \geq K_{t}\left(d_{1} d_{2}\right)^{\frac{\log r}{2 n \log |q|}}, \quad t=1,2,
$$

and

$$
\mu\left(f_{1}\right)+\mu\left(f_{2}\right) \geq \frac{\log d_{1}+\log d_{2}}{n \log |q|} .
$$

Example 1.1 The function $\left(f_{1}(z), f_{2}(z)\right)=\left(\frac{e^{z}}{z}, \frac{e^{z}}{-z}\right)$ satisfies the system of the form

$$
\left\{\begin{array}{l}
\sum_{j=1}^{n} \frac{2^{j} z+c_{j}}{e^{c_{j}} z^{2}} f_{1}\left(2^{j} z+c_{j}\right)=\sum_{j=1}^{n} f_{2}(z)^{2^{j}}, \\
\sum_{j=1}^{n} \frac{-\left(2^{j} z+c_{j}\right)}{e^{c_{j}} z^{j}} f_{2}\left(2^{j} z+c_{j}\right)=\sum_{j=1}^{n} f_{1}(z)^{2 j},
\end{array}\right.
$$

with rational coefficients, where $|q|=2>1, d_{1}=d_{2}=2^{n}$ and $c_{j} \in \mathbb{C}$. Since $n<2^{n}=d_{1}=d_{2}$ for all $n \in \mathbb{N}_{+}$, we have $\log M\left(r, f_{t}\right)=r-\log r \geq \frac{1}{2} r=\frac{1}{2}\left(d_{1} d_{2}\right)^{\frac{\log r}{2 n \log |q|}}(r \rightarrow \infty)$ and $\mu\left(f_{t}\right)=$ $\sigma\left(f_{t}\right)=1=\frac{\log \left(d_{1} d_{2}\right)}{2 n \log |q|}$ for $t=1,2$. This shows that the conclusion of Theorem 1.4 is sharp and the equality in the consequent result $\mu\left(f_{1}\right)+\mu\left(f_{2}\right) \geq \frac{2\left(\log d_{1}+\log d_{2}\right)}{\left(n_{1}+n_{2}\right) \log |q|}$ of Remark 1.1 can be arrived. 
Let $q, c_{j}$ be stated as in Theorem 1.5, set

$$
\begin{aligned}
& F_{1}\left(z ; f_{1}, n_{1}, q, c_{j}\right)=\frac{\sum_{\lambda^{1} \in I_{1}} d_{\lambda^{1}}(z) f_{1}\left(q z+c_{1}\right)^{i_{\lambda_{1}^{1}}} f_{1}\left(q^{2} z+c_{2}\right)^{i_{\lambda_{2}}^{1}} \cdots f_{1}\left(q^{n_{1}} z+c_{n_{1}}\right)^{i_{\lambda_{n_{1}}}}}{\sum_{\mu^{1} \in J_{1}} e_{\mu^{1}}(z) f_{1}\left(q z+c_{1}\right)^{j_{1}^{1}} f_{1}\left(q^{2} z+c_{2}\right)^{j_{2}} \cdots f_{1}\left(q^{n_{1}} z+c_{n_{1}}\right)^{\mu_{n_{1}}^{1}}}, \\
& F_{2}\left(z ; f_{2}, n_{2}, q, c_{j}\right)=\frac{\sum_{\lambda^{2} \in I_{2}} d_{\lambda^{2}}(z) f_{2}\left(q z+c_{1}\right)^{i \lambda_{1}^{2}} f_{2}\left(q^{2} z+c_{2}\right)^{i_{\lambda_{2}}^{2}} \cdots f_{2}\left(q^{n_{2}} z+c_{n_{2}}\right)^{i_{\lambda_{n_{2}}^{2}}}}{\sum_{\mu^{2} \in J_{2}} e_{\mu^{2}}(z) f_{2}\left(q z+c_{1}\right)^{j \mu_{1}^{2}} f_{2}\left(q^{2} z+c_{2}\right)^{j_{2}^{2}} \cdots f_{2}\left(q^{n_{2}} z+c_{n_{2}}\right)^{\mu_{n_{2}}^{2}}} .
\end{aligned}
$$

Now, we will investigate the lower order of meromorphic solutions of a type of system of complex function equations and obtain a result as follows.

Theorem 1.6 Suppose that $\left(f_{1}, f_{2}\right)$ are a pair of transcendental meromorphic solutions of the system of $q$-difference equations

$$
\left\{\begin{array}{l}
F_{1}\left(z ; f_{1}, n_{1}, q, c_{j}\right)=\frac{\sum_{j=0}^{s_{1}} a_{j}^{1}(z) f_{2}(z)^{j}}{\sum_{j=0}^{1} b_{j}^{1}(z) f_{2}(z)^{j}}, \\
F_{2}\left(z ; f_{2}, n_{2}, q, c_{j}\right)=\frac{\sum_{j=0}^{s_{2}} a_{j}^{2}(z) f_{1}(z)^{j}}{\sum_{j=0}^{l_{2}} b_{j}^{2}(z) f_{1}(z)^{\prime}},
\end{array}\right.
$$

where $I_{t}=\left\{\left(i_{\lambda_{1}^{t}}, i_{\lambda_{2}^{t}}, \ldots, i_{\lambda_{n_{t}}^{t}}\right)\right\}, J_{t}=\left\{j_{\mu_{1}^{t}}, j_{\mu_{2}^{t}}, \ldots, j_{\mu_{n_{t}}^{t}}\right\}$ are finite index sets satisfying

$$
\max _{\lambda^{t}, \mu^{t}}\left\{i_{\lambda_{1}^{t}}+i_{\lambda_{2}^{t}}+\cdots+i_{\lambda_{n_{t}}^{t}}, j_{\mu_{1}^{t}}+j_{\mu_{2}^{t}}+\cdots+j_{\mu_{n_{t}}^{t}}\right\}=\sigma_{t}, \quad t=1,2,
$$

and $d_{t}=\max \left\{s_{t}, l_{t}\right\} \geq 2, t=1,2$, and all coefficients of $(6)$ are of growth $S\left(r, f_{1}\right), S\left(r, f_{2}\right)$. If

$$
d_{1} d_{2}>4 n_{1} n_{2} \sigma_{1} \sigma_{2}
$$

then for sufficiently large $r$,

$$
T\left(r, f_{t}\right) \geq K_{t}\left(\frac{d_{1} d_{2}}{4 n_{1} n_{2} \sigma_{1} \sigma_{2}}\right)^{\frac{\log r}{\left(n_{1}+n_{2}\right) \log |q|}}, \quad t=1,2
$$

where $K_{t}>0$ are constants. Thus, the lower order of $f_{1}, f_{2}$ satisfy

$$
\mu\left(f_{1}\right)+\mu\left(f_{2}\right) \geq \frac{2\left(\log d_{1} d_{2}-\log 4 n_{1} n_{2} \sigma_{1} \sigma_{2}\right)}{\left(n_{1}+n_{2}\right) \log |q|} .
$$

Example 1.2 The functions $\left(f_{1}(z), f_{2}(z)\right)=\left(e^{z^{2}}, e^{-z^{2}}\right)$ satisfy the system of function equations

$$
\left\{\begin{array}{l}
\frac{f_{2}\left(4 z+c_{2}\right)+f_{2}\left(2 z+c_{1}\right) f_{2}\left(4 z+c_{2}\right)}{f_{2}\left(2 z+c_{1}\right)}=\frac{a_{1} f_{1}(z)^{4}+a_{0}}{f_{1}(z)^{16}}, \\
\frac{f_{1}\left(2 z+c_{1}\right)+f_{1}\left(2 z+c_{1}\right) f_{1}\left(4 z+c_{2}\right)}{f_{1}\left(4 z+c_{2}\right)}=\frac{b_{1} f_{2}(z)^{16}+b_{0}}{f_{2}(z)^{4}},
\end{array}\right.
$$

with small function coefficients

$$
a_{1}=e^{-8 z c_{2}-4 z c_{1}} e^{c_{2}^{2}-c_{1}^{2}}, \quad a_{0}=e^{-8 z c_{2}-c_{2}^{2}}, \quad b_{1}=e^{4 z c_{1}+c_{1}^{2}-8 z c_{2}-c_{2}^{2}}, \quad b_{0}=e^{4 z c_{1}+c_{1}^{2}},
$$


where $q=n_{1}=n_{2}=\sigma_{1}=\sigma_{2}=2, d_{1}=d_{2}=16, d_{1} d_{2}=256>4 n_{1} n_{2} \sigma_{1} \sigma_{2}, c_{1}, c_{2} \in \mathbb{C}$ and $a_{1}, a_{0}$, $b_{1}, b_{0}$ are small functions of $f_{1}, f_{2}$. We have $\mu\left(f_{t}\right)=\sigma\left(f_{t}\right)=2, t=1,2$ and

$$
\mu\left(f_{1}\right)+\mu\left(f_{2}\right)=4>1=\frac{2\left(\log d_{1} d_{2}-\log 4 n_{1} n_{2} \sigma_{1} \sigma_{2}\right)}{\left(n_{1}+n_{2}\right) \log |q|} .
$$

This shows that Theorem 1.6 may hold.

\section{The proof of Theorem 1.3}

Denote $G_{t}(z)=\prod_{j=1}^{n_{t}} f_{t}\left(z+c_{j}\right), t=1,2$. By applying Valiron-Mohon'ko theorem [22] to (2), we have

$$
\begin{aligned}
& T\left(r, G_{1}\right)=\max \left\{p_{2}, q_{2}\right\} T\left(r, f_{2}\right)+S\left(r, f_{1}\right)+S\left(r, f_{2}\right), \\
& T\left(r, G_{2}\right)=\max \left\{p_{1}, q_{1}\right\} T\left(r, f_{1}\right)+S\left(r, f_{1}\right)+S\left(r, f_{2}\right) .
\end{aligned}
$$

From (3), we can take constants $\xi_{t}, \delta_{t}$ such that

$$
\max \left\{\lambda\left(f_{t}\right), \lambda\left(1 / f_{t}\right)\right\}<\xi_{t}<\delta_{t}<\rho\left(f_{t}\right), \quad t=1,2,
$$

then we have

$$
T\left(r, \frac{f_{t}^{\prime}}{f_{t}}\right)=\bar{N}\left(r, f_{t}\right)+\bar{N}\left(r, \frac{1}{f_{t}}\right)+S\left(r, f_{t}\right)=O\left(r^{\xi t}\right)+S\left(r, f_{t}\right), \quad t=1,2 .
$$

From (8) and the definitions of $G_{t}(t=1,2)$, similar to the above argument, we have

$$
\begin{aligned}
T\left(r, \frac{G_{t}^{\prime}}{G_{t}}\right) & =N\left(r, \frac{G_{t}^{\prime}}{G_{t}}\right)+m\left(r, \frac{G_{t}^{\prime}}{G_{t}}\right) \\
& \leq n_{t} \bar{N}\left(r+C, f_{t}\right)+n_{t} \bar{N}\left(r, \frac{1}{f_{t}}\right)+S\left(r, f_{1}\right)+S\left(r, f_{2}\right) \\
& =O\left(r^{\xi t}\right)+S\left(r, f_{1}\right)+S\left(r, f_{2}\right),
\end{aligned}
$$

where $C:=\max \left\{\left|c_{i}\right|,\left|c_{j}\right|, i=1,2, \ldots, n_{1} ; j=1,2, \ldots, n_{2}\right\}$. From (3), we know that zeros and poles are Borel exceptions of $f_{t}(t=1,2)$, and from [23, Satz 13.4], we have that $f_{t}(t=1,2)$ is of regular growth. Hence, there exists $r_{0}>0$ such that $T\left(r, f_{t}\right)>r^{\delta_{t}}$ for $r>r_{0}$. So, we can get that

$$
T\left(r, \frac{G_{t}^{\prime}}{G_{t}}\right)=S\left(r, f_{1}\right)+S\left(r, f_{2}\right), \quad T\left(r, \frac{f_{t}^{\prime}}{f_{t}}\right)=S\left(r, f_{1}\right)+S\left(r, f_{2}\right), \quad t=1,2 .
$$

Now, we rewrite system (2) as

$$
\left\{\begin{array}{l}
\frac{b_{p_{2}}(z)}{a_{p_{2}}(z)} G_{1}(z)=\frac{P_{2}\left(z, f_{2}\right)}{Q_{2}\left(z, f_{2}\right)}=u_{2}\left(z, f_{2}\right), \\
\frac{d_{p_{1}}(z)}{e_{p_{1}}(z)} G_{2}(z)=\frac{P_{1}\left(z, f_{1}\right)}{Q_{1}\left(z, f_{1}\right)}=u_{1}\left(z, f_{1}\right),
\end{array}\right.
$$

without loss of generality, assume that $P_{t}, Q_{t}$ are monic polynomials in $f_{t}$ with coefficients of growth $S\left(r, f_{1}\right), S\left(r, f_{2}\right)$. Set $F_{t}:=\frac{f_{t}^{\prime}}{f_{t}}, U_{t}:=\frac{u_{t}^{\prime}}{u_{t}}, t=1,2$. From (9), we have $T\left(r, U_{t}\right)=$ 
$S\left(r, f_{1}\right)+S\left(r, f_{2}\right)$. And because

$$
\left\{\begin{array}{l}
\frac{P_{2}^{\prime} Q_{2}-P_{2} Q_{2}^{\prime}}{Q_{2}^{2}}=u_{2}^{\prime}=U_{2} u_{2}=\frac{U_{2} P_{2}}{Q_{2}} \\
\frac{P_{1}^{\prime} Q_{1}-P_{1} Q_{1}^{\prime}}{Q_{1}^{2}}=u_{1}^{\prime}=U_{1} u_{1}=\frac{U_{1} P_{1}}{Q_{1}}
\end{array}\right.
$$

it follows that

$$
\left\{\begin{array}{l}
P_{2}^{\prime} Q_{2}-P_{2} Q_{2}^{\prime}=U_{2} P_{2} Q_{2} \\
P_{1}^{\prime} Q_{1}-P_{1} Q_{1}^{\prime}=U_{1} P_{1} Q_{1}
\end{array}\right.
$$

Substituting $f_{t}^{\prime}=F_{t} f_{t}, t=1,2$, to the above equalities and comparing the leading coefficients, we can get

$$
\left(p_{t}-q_{t}\right) F_{t}=U_{t}, \quad t=1,2
$$

Solving the above equations, we get

$$
u_{t}=\pi_{t}\left(f_{t}(z)\right)^{p_{2}-q_{2}}, \quad \pi_{t} \in \mathbb{C}, t=1,2 .
$$

From (9) and (10), it follows that

$$
\left\{\begin{array}{l}
G_{1}(z)=\pi_{2} \frac{a_{p_{2}}(z)}{b_{p_{2}}(z)}\left(f_{2}(z)\right)^{p_{2}-q_{2}} \\
G_{2}(z)=\pi_{1} \frac{e_{p_{1}}(z)}{d_{p_{1}}(z)}\left(f_{1}(z)\right)^{p_{1}-q_{1}}
\end{array}\right.
$$

Thus, we complete the proof of Theorem 1.3.

\section{Proofs of Theorems 1.4 and 1.5}

\subsection{The proof of Theorem 1.4}

Because the coefficients $a_{j}^{t}(z), b_{i}^{t}(z)(t=1,2)$ are rational functions, we can rewrite (4) as follows:

$$
\left\{\begin{array}{l}
\sum_{j=1}^{n} A_{j}^{1}(z) f_{1}\left(q^{j} z+c_{j}\right)=\sum_{i=0}^{d_{1}} B_{i}^{1}(z) f_{2}(z)^{i} \\
\sum_{j=1}^{n} A_{j}^{2}(z) f_{2}\left(q^{j} z+c_{j}\right)=\sum_{i=0}^{d_{2}} B_{i}^{2}(z) f_{1}(z)^{i}
\end{array}\right.
$$

where the coefficients $A_{j}^{t}(z), B_{i}^{t}(z)(t=1,2)$ are polynomials. We will consider two cases as follows.

Case 1. Since $\left(f_{1}, f_{2}\right)$ are a pair of solutions of system (4) or (11) and $f_{t}, t=1,2$, are transcendental entire, set $p_{i}^{t}=\operatorname{deg} A_{j}^{t}(j=1,2, \ldots, n), q_{i}^{t}=\operatorname{deg} B_{i}^{t}\left(i=0,1, \ldots, d_{i}\right), t=1,2$, and $C:=\max \left\{\left|c_{i}\right|,\left|c_{j}\right|, i=1,2, \ldots, n_{1} ; j=1,2, \ldots, n_{2}\right\}$. Taking $m_{t}=\max \left\{p_{1}^{t}, \ldots, p_{n}^{t}\right\}+1$, and from $|q|>1$ and $M\left(r, f_{t}\left(q^{j} z+c_{j}\right)\right) \leq M\left(|q|^{j} r+\left|c_{j}\right|, f_{t}\right)$, we have that

$$
\left\{\begin{array}{l}
M\left(r, \sum_{i=0}^{d_{1}} B_{i}^{1}(z) f_{2}(z)^{i}\right)=M\left(r, \sum_{j=1}^{n} A_{j}^{1}(z) f_{1}\left(q^{j} z+c_{j}\right)\right) \leq n r^{m_{1}} M\left(|q|^{n} r+C, f_{1}\right), \\
M\left(r, \sum_{i=0}^{d_{2}} B_{i}^{2}(z) f_{1}(z)^{i}\right)=M\left(r, \sum_{j=1}^{n} A_{j}^{2}(z) f_{2}\left(q^{j} z+c_{j}\right)\right) \leq n r^{m_{2}} M\left(|q|^{n} r+C, f_{2}\right),
\end{array}\right.
$$

when $r$ is sufficiently large. Since $B_{i}^{t}\left(i=0,1, \ldots, d_{t} ; t=1,2\right)$ are polynomials and $f_{t}(t=1,2)$ are transcendental entire functions, we have $M\left(r, \sum_{i=0}^{d_{1}-1} B_{i}^{1} f_{2}(z)^{i}\right)=o\left(M\left(r, f_{2}(z)^{d_{1}}\right)\right)$ and 
$M\left(r, \sum_{i=0}^{d_{2}-1} B_{i}^{2} f_{1}(z)^{i}\right)=o\left(M\left(r, f_{1}(z)^{d_{2}}\right)\right)$. Then, for sufficiently large $r$, it follows that

$$
\left\{\begin{array}{l}
M\left(r, \sum_{i=0}^{d_{1}} B_{i}^{1}(z) f_{2}(z)^{i}\right) \geq \frac{1}{2} M\left(r, B_{d_{1}}^{1} f_{2}(z)^{d_{1}}\right) \\
M\left(r, \sum_{i=0}^{d_{2}} B_{i}^{2}(z) f_{1}(z)^{i}\right) \geq \frac{1}{2} M\left(r, B_{d_{2}}^{2} f_{1}(z)^{d_{2}}\right) .
\end{array}\right.
$$

From (12) and (13), for sufficiently large $r$ it follows that

$$
\left\{\begin{array}{l}
\log M\left(|q|^{n} r+C, f_{1}\right) \geq d_{1} \log M\left(r, f_{2}\right)+g_{1}(r) \\
\log M\left(|q|^{n} r+C, f_{2}\right) \geq d_{2} \log M\left(r, f_{1}\right)+g_{2}(r),
\end{array}\right.
$$

where $\left|g_{t}(r)\right|<K_{t} \log r, t=1,2$, for some constants $K_{t}>0$. From (14), for sufficiently large $r$, we get

$$
\log M\left(|q|^{2 n} r+C+C|q|^{n}, f_{1}\right) \geq d_{1} d_{2} \log M\left(r, f_{1}\right)+g_{1}\left(|q|^{n} r+C\right)+d_{1} g_{2}(r)
$$

Iterating (15), we have

$$
\log M\left(|q|^{2 n k} r+C \sum_{\nu=0}^{2 k-1}|q|^{\nu n}, f_{1}\right) \geq\left(d_{1} d_{2}\right)^{k} \log M\left(r, f_{1}\right)+E_{k}^{1}(r)+E_{k}^{2}(r) \quad(k \in \mathbb{N})
$$

where

$$
\begin{aligned}
\left|E_{k}^{1}(r)\right| & =\left|\left(d_{1} d_{2}\right)^{k-1} g_{1}\left(|q|^{n} r+C\right)+\cdots+g_{1}\left(|q|^{(2 k-1) n} r+C \sum_{\nu=0}^{2 k-2}|q|^{\nu n}\right)\right| \\
& \leq K_{1}\left(d_{1} d_{2}\right)^{k-1} \sum_{j=0}^{k-1} \frac{\log |q|^{(2 j+1) n} r+C \sum_{\nu=0}^{2 j-1} \log |q|^{v n}}{\left(d_{1} d_{2}\right)^{j}} \\
& \leq K_{1}\left(d_{1} d_{2}\right)^{k-1} \sum_{j=0}^{\infty} \frac{\log |q|^{(2 j+1) n} r+C \sum_{\nu=0}^{2 j-1} \log |q|^{v n}}{\left(d_{1} d_{2}\right)^{j}}
\end{aligned}
$$

and

$$
\begin{aligned}
\left|E_{k}^{2}(r)\right| & =\left|d_{1}\left(d_{1} d_{2}\right)^{k-1} g_{2}(r)+\cdots+d_{1} g_{2}\left(|q|^{(2 k-2) n} r+C \sum_{\nu=0}^{2 k-3}|q|^{v n}\right)\right| \\
& \leq K_{2} d_{1}\left(d_{1} d_{2}\right)^{k-1} \sum_{j=0}^{k-1} \frac{\log |q|^{2(j-1) n} r+C \sum_{\nu=0}^{2 j-3}|q|^{\nu n}}{\left(d_{1} d_{2}\right)^{j}} \\
& \leq K_{2} d_{1}\left(d_{1} d_{2}\right)^{k-1} \sum_{j=0}^{\infty} \frac{\log |q|^{2(j-1) n} r+C \sum_{\nu=0}^{2 j-3}|q|^{\nu n}}{\left(d_{1} d_{2}\right)^{j}} .
\end{aligned}
$$

Observe that $|q|>1$, then for sufficiently large $r$, we have

$$
\begin{aligned}
\log |q|^{(2 j+1) n} r+C \sum_{\nu=0}^{2 j}|q|^{\nu n} & \leq \log |q|^{(2 j+1) n}+\log r+\log (2 j+1) C+\log |q|^{2 j n} \\
& \leq 2 j(2 j+1) n^{2}(\log |q|)^{2} \log j \log C \log r .
\end{aligned}
$$


And since $d_{1} d_{2} \geq 2$, it follows that the series $\sum_{i=0}^{\infty} \frac{2 j(2 j+1) n^{2}\left(\left.\log |q|\right|^{2} \log j\right.}{\left(d_{1} d_{2}\right)^{i}}$ is convergent. Thus, for sufficiently large $r$, we have

$$
\left|E_{k}^{t}(r)\right| \leq K_{t}^{\prime}\left(d_{1} d_{2}\right)^{k} \log r, \quad t=1,2,
$$

where $K_{t}^{\prime}>0(t=1,2)$ are some constants. Since $f_{1}$ is a transcendental entire function, for sufficiently large $r$, we have

$$
\log M\left(r, f_{1}\right) \geq 3 K^{\prime} \log r
$$

where $K^{\prime}>\max \left\{K_{1}^{\prime}, K_{2}^{\prime}\right\}$. Hence, from (16)-(17), there exists $r_{0} \geq e$ such that for $r \geq r_{0}$, we have

$$
\log M\left(|q|^{2 n k} r+C \sum_{\nu=0}^{2 k-1}|q|^{\nu n}, f_{1}\right) \geq K^{\prime}\left(d_{1} d_{2}\right)^{k} \log r
$$

Thus, for each sufficiently large $R$, there exists $k \in \mathbb{N}$ such that

$$
R \in\left[|q|^{2 n k} r_{0}+C \sum_{\nu=0}^{2 k-1}|q|^{\nu n},|q|^{2 n(k+1)} r_{0}+C \sum_{\nu=0}^{2 k+1}|q|^{\nu n}\right),
$$

i.e.,

$$
k>\frac{\log R+\log \left(|q|^{n}-1\right)-\log r_{0}-\log C-4 n \log |q|}{2 n \log |q|} .
$$

From (19) and (20), we have

$$
\begin{aligned}
\log M\left(R, f_{1}\right) & \geq \log M\left(|q|^{2 n k} r_{0}+C \sum_{\nu=0}^{2 k-1}|q|^{\nu n}, f_{1}\right) \\
& \geq K^{\prime}\left(d_{1} d_{2}\right)^{k} \log r_{0} \\
& \geq K^{\prime \prime}\left(d_{1} d_{2}\right)^{\frac{\log R}{2 \log |q|}},
\end{aligned}
$$

where

$$
K^{\prime \prime}=K^{\prime}\left(d_{1} d_{2}\right)^{\frac{\log \left(|q|^{n}-1\right)-\log r_{0}-\log C-4 n \log |q|}{2 n \log |q|}} .
$$

Similar to the above argument, we can get that there exist constants $K>0$ and $r_{0}>0$ such that for all $r \geq r_{0}$,

$$
\log M\left(r, f_{2}\right) \geq K\left(d_{1} d_{2}\right)^{\frac{\log r}{2 n \log |q|}} .
$$

Case 2. Suppose that $\left(f_{1}, f_{2}\right)$ are a pair of solutions of system $(4)$ and $f_{t}(t=1,2)$ are meromorphic with finitely many poles. Then there exist polynomials $P_{t}(z)$ such that $g_{t}(z)=P_{t}(z) f_{t}(z)(t=1,2)$ are entire functions. Substituting $f_{t}(z)=\frac{g_{t}(z)}{P_{t}(z)}$ into (11) and again multiplying away the denominators, we can get a system similar to (11). By using the same 
argument as in the above, we can get that for sufficiently large $r$,

$$
\log M\left(r, f_{t}\right)=\log M\left(r, g_{t}\right)+O(1) \geq\left(K_{t}^{\prime \prime}-\varepsilon\right)\left(d_{1} d_{2}\right)^{\frac{\log r}{2 n \log |q|}} \geq K_{t}^{\prime \prime \prime}\left(d_{1} d_{2}\right)^{\frac{\log r}{2 \log |q|}}
$$

where $K_{t}^{\prime \prime \prime}(>0)(t=1,2)$ are some constants.

From Case 1 and Case 2, this completes the proof of Theorem 1.4.

\subsection{The proof of Theorem 1.5}

Since the coefficients of $P_{t}\left(z, f_{t}(z)\right), Q_{t}\left(z, f_{t}(z)\right)$ are rational functions, we can choose a sufficiently large constant $R(>0)$ such that the coefficients of $P_{t}\left(z, f_{t}(z)\right), Q_{t}\left(z, f_{t}(z)\right)(t=1,2)$ have no zeros or poles in $\{z \in \mathbb{C}:|z|>R\}$. Assume that $\left(f_{1}, f_{2}\right)$ is a solution of system (5) and $f_{t}(t=1,2)$ are transcendental, since $f_{t}(t=1,2)$ have infinitely many poles. Thus, without loss of generality, we choose a pole $z_{0}$ of $f_{1}$ of multiplicity $\mu \geq 1$ satisfying $\left|z_{0}\right|>R$. Since $d_{1}=s_{1}-t_{1} \geq 2$, then the right-hand side of the second equation in (5) has a pole of multiplicity $d_{1} \mu$ at $z_{0}$. Therefore, there exists at least one index $j_{1} \in\left\{1,2, \ldots, n_{2}\right\}$ such that $q^{j_{1}} z_{0}+c_{j_{1}}$ is a pole of $f_{2}$ of multiplicity $\mu_{1}^{\prime} \geq d_{1} \mu$. If $\left|q^{j_{1}} z_{0}+c_{j_{1}}\right| \leq R$, this process will be terminated and we have to choose another pole $z_{0}$ of $f_{1}$ in the way we did above. If $\left|q^{j_{1}} z_{0}+c_{j_{1}}\right|>R$, since $d_{2}=s_{2}-t_{2} \geq 2$, then the right-hand side of the first equation in (5) has a pole of multiplicity $d_{2} \mu_{1}^{\prime} \geq d_{1} d_{2} \mu$. Therefore, there exists at least one index $j_{1}^{\prime} \in\left\{1,2, \ldots, n_{1}\right\}$ such that $q^{i_{1}^{\prime}}\left(q^{j_{1}} z_{0}+c_{j_{1}}\right)+c_{j_{1}^{\prime}}$ is a pole of $f_{1}$ of multiplicity $\mu_{1} \geq d_{2} \mu_{1}^{\prime} \geq d_{1} d_{2} \mu$.

We proceed to follow the step above, we can get a sequence

$$
\left\{\zeta_{k}\right\}_{k=1}^{\infty}:=\left\{\prod_{i=1}^{k} q^{j_{i}+j_{i}^{\prime}} z_{0}+\sum_{s=1}^{k} \prod_{i=s+1}^{k} q^{j_{i}+j_{i}^{\prime}}\left(q^{j_{s}^{\prime}} c_{j_{s}}+c_{j_{s}^{\prime}}\right)\right\}_{k=1}^{\infty},
$$

where $\zeta_{k}$ is a pole of $f_{1}$ with multiplicity $\mu_{k}, j_{s} \in\left\{1,2, \ldots, n_{2}\right\}$ and $j_{s}^{\prime} \in\left\{1,2, \ldots, n_{1}\right\}$. From the above discussion, we can get $\mu_{k} \geq\left(d_{1} d_{2}\right)^{k} \mu$. Obviously, we have $\left|\zeta_{k}\right| \rightarrow \infty$ as $k \rightarrow \infty$. Then there exists a positive integer $k_{0} \in \mathbb{N}_{+}$such that for sufficiently large $k\left(\geq k_{0}\right)$,

$$
\begin{aligned}
\mu\left(d_{1} d_{2}\right)^{k} & \leq \mu\left[1+d_{1} d_{2}+\cdots+\left(d_{1} d_{2}\right)^{k}\right] \leq n\left(\left|\zeta_{k}\right|, f_{1}\right) \\
& \leq n\left(|q|^{\left(n_{1}+n_{2}\right) k}\left|z_{0}\right|+C\left(|q|^{n_{1}}+1\right) \sum_{i=0}^{k-1}|q|^{i\left(n_{1}+n_{2}\right)}, f_{1}\right),
\end{aligned}
$$

where $C:=\max \left\{\left|c_{i}\right|,\left|c_{j}\right|, i=1,2, \ldots, n_{1} ; j=1,2, \ldots, n_{2}\right\}$. Thus, for each sufficiently large $r$, there exists $k \in \mathbb{N}_{+}$such that $r \in\left[\eta_{k}, \eta_{k+1}\right)$, where $\eta_{k}:=|q|^{\left(n_{1}+n_{2}\right) k}\left|z_{0}\right|+C\left(|q|^{n_{1}}+1\right) \times$ $\sum_{i=0}^{k-1}|q|^{i\left(n_{1}+n_{2}\right)}$, it follows that

$$
k>\frac{\log r-\log \left|z_{0}\right|-\log C-\log \left(|q|^{n_{1}}+1\right)-\left(n_{1}+n_{2}\right) \log |q|+\log \left(|q|^{n_{1}+n_{2}}-1\right)}{\left(n_{1}+n_{2}\right) \log |q|} .
$$

From (23) and (24), we have

$$
\begin{aligned}
n\left(r, f_{1}\right) & \geq \mu\left(d_{1} d_{2}\right)^{k} \geq \mu\left(d_{1} d_{2}\right)^{\frac{\log r-\log \left|z_{0}\right|-\log C-\log \left(|q|^{n_{1}}+1\right)-\left(n_{1}+n_{2}\right) \log |q|+\log \left(|q|^{\left.n_{1}+n_{2}-1\right)}\right.}{\left(n_{1}+n_{2}\right) \log |q|}} \\
& \geq K_{5} d^{\frac{\log r}{\left(n_{1}+n_{2}\right) \log |q|}}
\end{aligned}
$$


where

$$
K_{5}=\mu\left(d_{1} d_{2}\right) \frac{\frac{-\log \left|z_{0}\right|-\log C-\log \left(|q|^{n_{1}}+1\right)-\left(n_{1}+n_{2}\right) \log |q|+\log \left(|q|^{\left.n_{1}+n_{2}-1\right)}\right.}{\left(n_{1}+n_{2}\right) \log |q|}}{.}
$$

And there exists $r_{0}>0$ and for all $r \geq r_{0}$, we have

$$
K_{5}\left(d_{1} d_{2}\right)^{\frac{\log r}{\left(n_{1}+n_{2}\right) \log |q|}} \leq n\left(r, f_{1}\right) \leq \frac{1}{\log 2} T\left(2 r, f_{1}\right) .
$$

Similar to the above discussion, we can get that there exists $r_{0}>0$ and for all $r \geq r_{0}$, we have

$$
K_{5}^{\prime}\left(d_{1} d_{2}\right)^{\frac{\log r}{\left(n_{1}+n_{2}\right) \log |q|}} \leq n\left(r, f_{2}\right) \leq \frac{1}{\log 2} T\left(2 r, f_{2}\right) .
$$

From these inequalities, we can get $\mu\left(f_{1}\right)+\mu\left(f_{2}\right) \geq \frac{2\left(\log d_{1}+\log d_{2}\right)}{\left(n_{1}+n_{2}\right) \log |q|}$ easily.

Thus, the proof of Theorem 1.5 is completed.

\section{Proof of Theorem 1.6}

Lemma 4.1 [21, Lemma 2] Let $f_{1}, f_{2}, \ldots, f_{n}$ be meromorphic functions. Then

$$
T\left(r, \sum_{\lambda \in I} f_{1}^{i_{\lambda_{1}}} f_{2}^{i_{\lambda_{2}}} \cdots f_{n}^{i_{\lambda_{n}}}\right) \leq \sigma \sum_{i=1}^{n} T\left(r, f_{i}\right)+\log s
$$

where $I=\left\{i_{\lambda_{1}}, i_{\lambda_{2}}, \ldots, i_{\lambda_{n}}\right\}$ is an index set consisting of s elements, and $\sigma=\max _{\lambda \in I}\left\{i_{\lambda_{1}}+i_{\lambda_{2}}+\right.$ $\left.\cdots+i_{\lambda_{n}}\right\}$.

Proof of Theorem 1.6 From $|q|>1, c_{j} \in \mathbb{C}$ and [24, p.249], we have $T\left(r, f_{t}\left(q^{j} z+c_{j}\right)\right)=$ $T\left(|q|^{j} r+\left|c_{j}\right|, f_{t}\right)+O(1), t=1,2$. For any given $\varepsilon\left(0<\varepsilon<\frac{\sqrt{d_{1} d_{2}}-\sqrt{4 n_{1} n_{2} \sigma_{1} \sigma_{2}}}{\sqrt{d_{1} d_{2}}+\sqrt{4 n_{1} n_{2} \sigma_{1} \sigma_{2}}}\right)$, applying ValironMohon'ko theorem [22] and Lemma 4.1 to (6), it follows that

$$
\left\{\begin{aligned}
d_{1}(1-\varepsilon) T\left(r, f_{2}\right) & \leq d_{1} T\left(r, f_{2}\right)+S\left(r, f_{2}\right) \leq 2 \sigma_{1} \sum_{j=1}^{n_{1}} T\left(|q|^{j} r+C, f_{1}\right)+S\left(r, f_{1}\right) \\
& \leq 2 n_{1} \sigma_{1}(1+\varepsilon) T\left(|q|^{n_{1}} r+C, f_{1}\right), \\
d_{2}(1-\varepsilon) T\left(r, f_{1}\right) & \leq d_{2} T\left(r, f_{1}\right)+S\left(r, f_{1}\right) \leq 2 \sigma_{2} \sum_{j=1}^{n_{2}} T\left(|q|^{j} r+C, f_{2}\right)+S\left(r, f_{2}\right) \\
& \leq 2 n_{2} \sigma_{2}(1+\varepsilon) T\left(|q|^{n_{2}} r+C, f_{2}\right),
\end{aligned}\right.
$$

outside of a possible exceptional set of finite linear measure. Then from (25) there exists $r_{0}>0$ such that

$$
\left\{\begin{array}{l}
T\left(|q|^{n_{1}+n_{2}} r+C\left(|q|^{n_{1}}+1\right), f_{1}\right) \geq \frac{d_{1} d_{2}(1-\varepsilon)^{2}}{4 n_{1} n_{2} \sigma_{1} \sigma_{2}(1+\varepsilon)^{2}} T\left(r, f_{1}\right), \\
T\left(|q|^{n_{1}+n_{2}} r+C\left(|q|^{n_{2}}+1\right), f_{2}\right) \geq \frac{d_{1} d_{2}(1-\varepsilon)^{2}}{4 n_{1} n_{2} \sigma_{1} \sigma_{2}(1+\varepsilon)^{2}} T\left(r, f_{2}\right),
\end{array}\right.
$$

holds for all $r>r_{0}$. Iterating (26), for any $k \in \mathbb{N}_{+}$and $r \geq r_{0}$, we have

$$
\left\{\begin{array}{l}
T\left(|q|^{\left(n_{1}+n_{2}\right) k} r+C\left(|q|^{n_{1}}+1\right) \sum_{i=0}^{k-1}|q|^{i\left(n_{1}+n_{2}\right)}, f_{1}\right) \geq\left(\frac{d_{1} d_{2}(1-\varepsilon)^{2}}{4 n_{1} n_{2} \sigma_{1} \sigma_{2}(1+\varepsilon)^{2}}\right)^{k} T\left(r, f_{1}\right), \\
T\left(|q|^{\left(n_{1}+n_{2}\right) k} r+C\left(|q|^{n_{2}}+1\right) \sum_{i=0}^{k-1}|q|^{i\left(n_{1}+n_{2}\right)}, f_{2}\right) \geq\left(\frac{d_{1} d_{2}(1-\varepsilon)^{2}}{4 n_{1} n_{2} \sigma_{1} \sigma_{2}(1+\varepsilon)^{2}}\right)^{k} T\left(r, f_{2}\right) .
\end{array}\right.
$$


By employing the same argument as in the proof of Theorem 1.5, for sufficiently large $\varrho$, from the above inequalities, we can get

$$
\left\{\begin{array}{l}
T\left(\varrho, f_{1}\right) \geq K_{6} T\left(r_{0}, f_{1}\right)\left(\frac{d_{1} d_{2}(1-\varepsilon)^{2}}{4 n_{1} n_{2} \sigma_{1} \sigma_{2}(1+\varepsilon)^{2}}\right)^{\frac{\log \varrho}{\left.n_{1}+n_{2}\right) \log |q|}}, \\
T\left(\varrho, f_{2}\right) \geq K_{6}^{\prime} T\left(r_{0}, f_{2}\right)\left(\frac{d_{1} d_{2}(1-\varepsilon)^{2}}{4 n_{1} n_{2} \sigma_{1} \sigma_{2}(1+\varepsilon)^{2}}\right)^{\frac{\log \varrho}{\left(n_{1}+n_{2}\right) \log |q|}},
\end{array}\right.
$$

where

$$
\begin{aligned}
& K_{6}=\left(\frac{d_{1} d_{2}(1-\varepsilon)^{2}}{4 n_{1} n_{2} \sigma_{1} \sigma_{2}(1+\varepsilon)^{2}}\right)^{\frac{-\log \left|z_{0}\right|-\log C-\log \left(|q|^{n_{1}}+1\right)-\left(n_{1}+n_{2}\right) \log |q|+\log \mid\left(\left.||^{n}\right|^{\left.n+n_{2}-1\right)}\right.}{\left(n_{1}+n_{2}\right) \log |q|}}, \\
& K_{6}^{\prime}=\left(\frac{d_{1} d_{2}(1-\varepsilon)^{2}}{4 n_{1} n_{2} \sigma_{1} \sigma_{2}(1+\varepsilon)^{2}}\right)^{\frac{-\log \left|z_{0}\right|-\log C-\log \left(|q|^{n}+1\right)-\left(n_{1}+n_{2}\right) \log |q|+\log \mid\left(|q|^{\left.n_{1}+n_{2}-1\right)}\right.}{\left(n_{1}+n_{2}\right) \log |q|}} .
\end{aligned}
$$

Letting $\varepsilon \rightarrow 0$, from (27) we have

$$
\left\{\begin{array}{l}
T\left(\varrho, f_{1}\right) \geq K_{7}\left(\frac{d_{1} d_{2}}{4 n_{1} 1_{2} \sigma_{1} \sigma_{2}}\right)^{\frac{\log \varrho}{\left(n_{1}+n_{2}\right) \log |q|}}, \\
T\left(\varrho, f_{2}\right) \geq K_{7}^{\prime}\left(\frac{d_{1} d_{2}}{4 n_{1} n_{2} \sigma_{1} \sigma_{2}}\right)^{\frac{\log \varrho}{\left(n_{1}+n_{2}\right) \log |q|}}
\end{array}\right.
$$

where $K_{6}, K_{6}^{\prime}$ are constants satisfying

$$
\begin{aligned}
& K_{7}=T\left(r_{0}, f_{1}\right)\left(\frac{d_{1} d_{2}}{4 n_{1} n_{2} \sigma_{1} \sigma_{2}}\right)^{\frac{-\log \left|z_{0}\right|-\log C-\log \left(|q|^{n_{1}}+1\right)-\left(n_{1}+n_{2}\right) \log |q|+\left.\log || q\right|^{\left.n_{1}+n_{2}-1\right)}}{\left(n_{1}+n_{2}\right) \log |q|}}, \\
& K_{7}^{\prime}=T\left(r_{0}, f_{2}\right)\left(\frac{d_{1} d_{2}}{4 n_{1} n_{2} \sigma_{1} \sigma_{2}}\right)^{\frac{-\log \left|z_{0}\right|-\log C-\log \left(|q|^{n_{2}}+1\right)-\left(n_{1}+n_{2}\right) \log |q|+\log \left(|q|^{\left.n_{1}+n_{2}-1\right)}\right.}{\left(n_{1}+n_{2}\right) \log |q|}} .
\end{aligned}
$$

Thus, from (28) the lower order of $f_{1}, f_{2}$ satisfy

$$
\mu\left(f_{1}\right)+\mu\left(f_{2}\right) \geq \frac{2 \log \left(d_{1} d_{2}\right)-2 \log \left(4 n_{1} n_{2} \sigma_{1} \sigma_{2}\right)}{\left(n_{1}+n_{2}\right) \log |q|} .
$$

Hence, we complete the proof of Theorem 1.6.

\section{Competing interests}

The authors declare that they have no competing interests.

\section{Authors' contributions}

HW, HYX completed the main part of this article, HW, YH, HYX corrected the main theorems. All authors read and approved the final manuscript.

\section{Author details}

'Department of Informatics and Engineering, Jingdezhen Ceramic Institute, Jingdezhen, Jiangxi 333403, China. ${ }^{2}$ College of Business, Jingdezhen Ceramic Institute, Jingdezhen, Jiangxi 333403, China.

\section{Acknowledgements}

The authors thank the referees for their valuable suggestions to improve the present article. The first author was supported by the NNSF of China (61202313), the Natural Science Foundation of Jiang-Xi Province in China (2010GQS0119 and 20132BAB211001). 


\section{References}

1. Hayman, WK: Meromorphic Functions. Clarendon, Oxford (1964)

2. Yang, L: Value Distribution Theory. Springer, Berlin (1993)

3. Yi, HX, Yang, CC: Uniqueness Theory of Meromorphic Functions. Kluwer Academic, Dordrecht (2003). Chinese original: Science Press, Beijing (1995)

4. Chen, ZX, Huang, ZB, Zheng, XM: On properties of difference polynomials. Acta Math. Sci. 31B(2), 627-633 (2011)

5. Halburd, RG, Korhonen, RJ: Nevanlinna theory for the difference operator. Ann. Acad. Sci. Fenn., Math. 31, 463-478 (2006)

6. Heittokangas, J, Korhonen, RJ, Laine, I, Rieppo, J, Zhang, JL: Value sharing results for shifts of meromorphic functions, and sufficient conditions for periodicity. J. Math. Anal. Appl. 355, 352-363 (2009)

7. Laine, I, Yang, CC: Value distribution of difference polynomials. Proc. Jpn. Acad., Ser. A, Math. Sci. 83, 148-151 (2007)

8. Liu, K, Yang, LZ: Value distribution of the difference operator. Arch. Math. 92, 270-278 (2009)

9. Zhang, JL, Korhonen, RJ: On the Nevanlinna characteristic of $f(q z)$ and its applications. J. Math. Anal. Appl. 369, 537-544 (2010)

10. Barnett, DC, Halburd, RG, Korhonen, RJ, Morgan, W: Nevanlinna theory for the q-difference operator and meromorphic solutions of q-difference equations. Proc. R. Soc. Edinb., Sect. A, Math. 137, 457-474 (2007)

11. Chiang, YM, Feng, SJ: On the Nevanlinna characteristic of $f(z+\eta)$ and difference equations in the complex plane. Ramanujan J. 16, 105-129 (2008)

12. Halburd, RG, Korhonen, RJ: Difference analogue of the lemma on the logarithmic derivative with applications to difference equations. J. Math. Anal. Appl. 314, 477-487 (2006)

13. Silvennoinen, $\mathrm{H}$ : Meromorphic solutions of some composite functional equations. Ann. Acad. Sci. Fenn., Math. Diss. $13,14-20(2003)$

14. Gao, LY: On meromorphic solutions of a type of system of composite functional equations. Acta Math. Sci. 32B(2) 800-806 (2012)

15. Gao, LY: Systems of complex difference equations of Malmquist type. Acta Math. Sin. 55, 293-300 (2012)

16. Gao, LY: Estimates of $N$-function and $m$-function of meromorphic solutions of systems of complex difference equations. Acta Math. Sci. 32B(4), 1495-1502 (2012)

17. $\mathrm{Xu}, \mathrm{HY}, \mathrm{Liu}, \mathrm{BX}$, Tang, KZ: Some properties of meromorphic solutions of systems of complex $q$-shift difference equations. Abstr. Appl. Anal. 2013, Article ID 680956 (2013)

18. Xu, HY, Cao, TB, Liu, BX: The growth of solutions of systems of complex q-shift difference equations. Adv. Differ. Equ. $2012,216(2012)$

19. Xu, HY, Xuan, ZX: Some properties of solutions of a class of systems of complex $q$-shift difference equations. Adv. Differ. Equ. 2013, 271 (2013)

20. Heittokangas, J, Korhonen, RJ, Laine, I, Rieppo, J, Tohge, K: Complex difference equations of Malmquist type. Comput. Methods Funct. Theory 1(1), 27-39 (2001)

21. Zheng, XM, Chen, ZX: Some properties of meromorphic solutions of q-difference equations. J. Math. Anal. Appl. 361, 472-480 (2010)

22. Laine, I: Nevanlinna Theory and Complex Differential Equations. de Gruyter, Berlin (1993)

23. Jank, G, Volkmann, L: Einführung in die Theorie der ganzen und meromorphen Funktionen mit Anwendungen auf Diffrentialgleichungen. Birkhäuser, Basel (1985)

24. Bergweiler, W, Ishizaki, K, Yanagihara, N: Meromorphic solutions of some functional equations. Methods Appl. Anal. 5(3), 248-259 (1998) Correction: Methods Appl. Anal. 6(4), 617-618 (1999)

10.1186/1687-1847-2013-378

Cite this article as: Wang et al.: Growth and poles of solutions of systems of complex composite functional

equations. Advances in Difference Equations 2013, 2013:378

\section{Submit your manuscript to a SpringerOpen ${ }^{\circ}$ journal and benefit from:}

- Convenient online submission

Rigorous peer review

- Immediate publication on acceptance

- Open access: articles freely available online

- High visibility within the field

- Retaining the copyright to your article

Submit your next manuscript at $>$ springeropen.com 